\title{
Asymptotic Distribution of Probabilities of Misclassification for Edgeworth Series Distribution (ESD)
}

\author{
Awogbemi Clement Adeyeye \\ Statistics Department, National Mathematical Centre, Abuja, Nigeria \\ Email address: \\ awogbemiadeyeye@yahoo.com

\section{To cite this article:} \\ Awogbemi Clement Adeyeye. Asymptotic Distribution of Probabilities of Misclassification for Edgeworth Series Distribution (ESD). \\ Engineering Mathematics. Vol. 4, No. 1, 2020, pp. 1-9. doi: 10.11648/j.engmath.20200401.11
}

Received: October 16, 2019; Accepted: November 12, 2019; Published: May 28, 2020

\begin{abstract}
The exact distribution of the test statistics in multivariate case is quite complicated in many situations, even when the underlying distribution is multivariate normal. This is due to the complex nature of the expression and therefore, there is a need to derive the asymptotic expression for the distribution. In this study, the asymptotic distribution of errors of misclassification for Edgeworth Series is derived by using Taylor's expansion. The error of misclassification for the conditional probability of misclassification was expanded around the means emanating from populations one and two using approximated mean and variance of the errors of misclassification. The distribution of error of misclassification of the conditional probability of misclassification for ESD is approximately normal with mean zero and variance one.
\end{abstract}

Keywords: Asymptotic Distribution, Probability of Misclassification, Edgeworth Series Distribution, Approximate Mean, Approximate Variance

\section{Introduction}

Edgeworth Series Distribution (ESD) constitutes an expansion which is a series that approximates a probability distribution in terms of its cumulants and the Hermite polynomials. It relates the probability density function to that of a standard normal distribution [14]. The use of ESD is expedient because approximations to distribution of sample statistics of higher order than $n^{-\frac{1}{2}}$ is of concern interest in asymptotic theory of statistics. An important tool that evaluates the refinements is provided for by ESD. Its expansions take cognizance of a method of using information about a higher order moment to increase accuracy of approximation [12].

The distribution of the conditional probability of misclassification of Edgeworth Series Distribution (ESD) is intractable due to the complex nature of the expression [16]. It comprises of the normal density function, the cumulative normal distribution function and the Chebyshev's Hermite polynomial. In lieu of the aforementioned, the asymptotic distribution of the error of misclassification is obtained in this study by the Taylor series expansion and the approximate mean and variance of the error of misclassification is then evaluated.

The validity of the one-term Edgeworth expansion for the multivariate mean of a random sample drawn without replacement was shown, under a limiting non-latticeness condition on the population [1]. The theorem was then applied to deduce the one term expansion for the univariate statistics which was expressed in linear and quadratic forms. The results were applied to the theory of bootstrap to prove a one- term expansion in univariate lattice case.

A Bayesian Edgeworth expansion by Stein's identity was considered [14]. He derived an expansion for posterior distributions by using Stein's Identity and Hermite polynomials as techniques. Two examples were shown where the incorporation of the expansion and numerical integration produced reasonable approximations when the sample size is small. It was observed that there are no guidelines or methodologies for how many terms should be included in the expansion based on real data.

Edgeworth series expansion and the saddle point method were investigated [13]. This is to estimate the distribution function for the standardized mean of independent and identically distributed random variables. The saddle point method was applied to chance game by simulating the game, and then comparing the empirical distribution function with 
that of saddle point method. The saddle point method gave better estimation than the normal approximation at the discontinuity points where distribution function has jumps.

Finite sample approximations for the distribution functions of Generalized Empirical Likelihood (GEL) were derived, and the analytical results obtained were applied to estimators which serve as alternatives to Generalized Method of Moment (GMM) [7]. The simulation results for confidence interval construction indicated that higher order analytical correction work well compared to first order approximations and improve inferences in small samples.

Seven asymptotic error rate expansions for the sample linear discriminant function with the aim of determining how well the assignment rules performed by Ekezie and Onyeagu [4]. They considered the following asymptotic expansions using binary variables: Deev's asymptotic expansion, Efron's asymptotic expansion, Raudy's asymptotic expansion, Okamato's asymptotic expansion, Sayre's asymptotic expansion and Kharin's asymptotic expansion. Each of the asymptotic expansions was evaluated at 225 configurations of $\mathrm{n}, \mathrm{r}$, and $\mathrm{d}$; where $\mathrm{n}$ is sample size, $\mathrm{r}$ is the number of variables and $d=P_{2 j}-P_{1 j} \geq 0, j=1,2, \ldots r ; P_{i j}=\sum S_{j} \frac{n_{j}(x)}{n_{i}}$ and $S_{j}$ is the set of all patterns X with $X_{j}=1$. At each of the 225 configurations, the asymptotic expansion that has minimum variance is declared the best asymptotic expansion. Simulation results showed that the best in terms of minimum variance was Anderson's expansion. The expected value of expansion was reasonably good for both small and large sample sizes.

Edgeworth expansion up to orders 4 and 6 was used to model convolutional noise probability density function. An appropriate Lagrangean multiplier was derived to obtain new closed form approximated expressions for the conditional expectation and Mean Square Error (MSE) as a by product. Simulation results did not show any equalization improvement on Edgeworth expansion up to order 4 when using optimal Lagrangean multiplier over a non-optimal set [6].

The derivation of the validity of Edgeworth expansions for realized volatility estimator was justified, and a new optimal non-lattice distribution to ensure second order correctness of bootstrap was later proposed [15]. The Monte not Carlo simulations showed that the intervals based on the Edgeworth correction, improved the finite sample properties relative to the conventional intervals based on the normal approximation.

Asymptotic expansions for the Stirling of the first type were generated and also in the generalized form of Ewens or Karamata- Stirling distribution [18]. On the basis of the expansions, they developed some new results on the asymptotic properties of the mode and the maximum of the Stirling numbers and Ewens distribution. They concluded by proving that the mode is the nearest integer to $a_{n}$ for a set of n's of asymptotic density.

Edgeworth expansions of statistic based on the distribution of random variable on extreme spacing statistics was studied by [11]. A random variable $\mathrm{R}_{\mathrm{n}}$ is an extreme spacing statistic when $f(x)=\left\{1\left(x \leq \alpha_{n}\right)+1\left(x \leq \beta_{n}\right)\right\}$, where 1 (A) is an indicator function, $\alpha_{n}$ and $\beta_{n}$ are constants. The distribution of the extreme spacing statistics was approximated by two well known Edgeworth expansions denoted by:

$$
F_{n}^{*}(x)=\Phi(x)-\phi(x)\left(\frac{10^{-4}}{n}\right)\left[\frac{\sqrt{n}}{297}\left(1-x^{2}\right)+1082\left(x^{3}-3 x\right)+8\left(x^{5}-10 x^{3}+15 x\right)\right]
$$

And

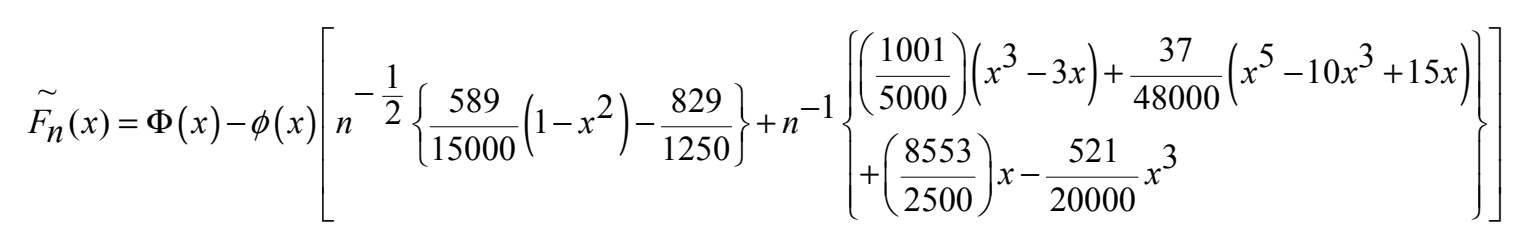

proposed by [3] and [5] respectively for different sample sizes. The symbols $\Phi(x)$ and $\phi(x)$ represent standard normal distribution and normal density function respectively. For the purpose of comparison, the values of the two expansions were simulated in the region $|x| \leq 3$, and it was observed that $\tilde{F}_{n}(x)$ performed better than $F_{n}{ }^{*}(x)$.

Although a lot of work has been done using Edgeworth Series expansion, the main objective of this research work is anchored on the derivation of the asymptotic distribution of probabilities of misclassification for Edgeworth Series Distribution.

\section{Probability Density Function of Edgeworth Series Distribution (ESD)}

Let $\mathrm{F}(\mathrm{x})$ be the distribution to be approximated, $\left\{k_{n}\right\}$ its cumulants, $\gamma_{k}$ the cumulants of a standard normal distribution function and $\mathrm{D}$ the differential operator with respect to $\mathrm{x}$. Also, let $\Phi$ and $\phi$ be the standard normal distribution and standard normal density function respectively. Then 


$$
\mathrm{F}(\mathrm{x})=\exp \sum_{n=1}^{\infty}\left(k_{n}-\gamma_{n}\right) \frac{(-D)^{n}}{\mathrm{n} !} \Phi_{(x)}
$$

This is identical with the expansions in Hermite orthogonal function for a probability density function

$$
P(x)=\sum_{n=0}^{\infty} c_{k} H_{n}(\mathrm{x}) \phi(x)
$$

where $H_{(n)}$ are Hermite polynomials and

$$
\begin{aligned}
& C_{k}=\frac{1}{k !} \int_{-\infty}^{\infty} P(x) H_{(n)}(x) d x \\
& P\left(\frac{n^{\frac{1}{2}}\left(\hat{\theta}_{n}-\theta_{0}\right)}{\sigma} \leq x\right)=\Phi(x)+n^{-\frac{1}{2}} P_{1}(x) \phi(x)+\ldots+\mathrm{n}^{-\frac{j}{2}} P_{j}(x) \phi(x)+\ldots
\end{aligned}
$$

where $\phi(x)=\sigma^{-1}(2 \pi)^{-\frac{1}{2}} e^{-\frac{x^{2}}{2 \sigma^{2}}}$ is the standard normal density function and $\Phi(x)=\int_{-\infty}^{x} \phi(x) d u$ is the standard normal distribution function Eq. (4) is the Edgeworth Series expansion. The functions $P_{j}$ are polynomials with coefficients depending on cumulants of $\hat{\theta}_{n}-\theta_{0}$. In particular, $P_{j}$ is a polynomial of degree at $3 \mathrm{j}-1$.

Suppose $\quad X_{i j}, i=1,2, j=1,2, \ldots, n_{i} \quad$ denote two independent random samples from populations $\pi_{i}, i=1,2$ respectively. The observations $X_{i j}$ emanate from the common distribution defined by the density function

$$
f_{i}(x)=\left(1-\frac{\lambda_{3}}{6} D^{3}\right) \phi\left(\frac{x-\mu_{i}}{\sigma}\right),-\infty<x<\infty \quad i=1,2
$$

The parameter $\lambda_{3}, \mu_{i}(i=1,2)$ satisfies the conditions: $-\infty<\lambda_{3}<\infty,-\infty<\mu_{i}<\infty$ and $\sigma>0$, where $D$ denotes the differential operator $\frac{d}{d x}$,

$$
\begin{aligned}
f\left(X_{0}+p, Y_{0}+q\right) & =f\left(X_{0}, Y_{0}\right)+\left[p \frac{\partial}{\partial X}+q \frac{\partial}{\partial Y}\right] f\left(X_{0}, Y_{0}\right)+\frac{1}{2 !}\left[p \frac{\partial}{\partial X}+q \frac{\partial}{\partial Y}\right]^{2} f\left(X_{0}, Y_{0}\right) \\
& +\ldots+\frac{1}{n !}\left[p \frac{\partial}{\partial X}+q \frac{\partial}{\partial Y}\right]^{n} f\left(X_{0}, Y_{0}\right)+G_{n}
\end{aligned}
$$

where $G_{n}$ is the remainder after $n$ terms and is given by 


$$
G_{n}=\frac{1}{(n+1) !}\left(p \frac{\partial}{\partial X}+q \frac{\partial}{\partial Y}\right)^{n+1} f\left(X_{0}+\theta p, Y_{0}+\theta q\right), 0<\theta<1
$$

Let $\alpha_{1}[\widehat{R}, f]$ and $\alpha_{2}[\widehat{R}, f]$ be the respective conditional probability of misclassification when an observation $\mathrm{X}$ from each of the populations $\pi_{1}$ and $\pi_{2}$ and is misclassified.

From above, $\alpha_{1}[\hat{R}, f]$ is a function of $\bar{X}_{1}$ and $\bar{X}_{2}$, we set

$$
\alpha_{1}[\hat{R}, f]=e_{12}\left(\bar{X}_{1}, \bar{X}_{2}\right)
$$

and

$$
\alpha_{2}[\hat{R}, f]=e_{21}\left(\bar{X}_{1}, \bar{X}_{2}\right)
$$

The expansion of $e_{12}\left(\bar{X}_{1}, \bar{X}_{2}\right)$ around $\left(\mu_{1}, \mu_{2}\right)$ is given by

$$
\begin{aligned}
e_{12}\left(\bar{X}_{1}, \bar{X}_{2}\right)= & e_{12}\left(\mu_{1}-\mu_{2}\right)+\left[\left(\bar{X}_{1}-\mu_{1}\right) \frac{\partial}{\partial \bar{x}_{1}}+\left(\bar{X}_{2}-\mu_{2}\right) \frac{\partial}{\partial \bar{x}_{2}}\right] \mu_{1} \mu_{2} e_{12}\left(\bar{X}_{1}, \mu_{1}\right) \\
& +\frac{1}{2 !}\left[\left(\bar{X}_{1}-\mu_{1}\right) \frac{\partial}{\partial \bar{x}_{1}}+\left(\bar{X}_{2}-\mu_{2}\right) \frac{\partial}{\partial \bar{x}_{2}}\right]^{2} \mu_{1}, \mu_{2}, e_{12}\left(\bar{X}_{1}, \bar{X}_{2}\right), \ldots \\
& +\frac{1}{n !}\left[\left(\bar{X}_{1}-\mu_{1}\right) \frac{\partial}{\partial \bar{x}_{1}}+\left(\bar{X}_{2}-\mu_{1}\right) \frac{\partial}{\partial \bar{x}_{2}}\right]^{n} \mu_{1}, \mu_{2}, e_{12}\left(\bar{X}_{1}, \bar{X}_{2}\right)+G_{n}
\end{aligned}
$$

While we consider the case when $\bar{X}_{1}<\bar{X}_{2}$,

$$
e_{12}\left(\bar{X}_{1}, \bar{X}_{2}\right)=\phi\left[\frac{2 \mu_{1}-\bar{X}_{1}-\bar{X}_{2}}{2 \sigma}\right]+\frac{\lambda_{3}}{6 \sigma^{2}} D^{2} \phi\left[\frac{\bar{X}_{1}+\bar{X}_{2}-2 \mu}{2 \sigma}\right]
$$

The partial derivatives of equation (9) with respect to $\bar{X}_{1}, \overline{\mathrm{X}}_{2}$ are given as

$$
\begin{gathered}
\frac{\partial}{\partial \bar{X}_{1}}\left[e_{12}\left(\bar{X}_{1}, \bar{X}_{2}\right)\right]=-\frac{1}{2 \sigma} \phi\left(\frac{2 \mu_{1}-\bar{X}_{1}-\bar{X}_{2}}{2 \sigma}\right) \\
+\frac{\lambda_{3}}{12 \sigma^{3}} D^{3} \phi\left(\frac{\bar{X}_{1}+\bar{X}_{2}-2 \mu_{1}}{2 \sigma}\right) . \\
\frac{\partial^{2}}{\partial \bar{X}_{1}}\left[e_{12}\left(\bar{X}_{1}, \bar{X}_{2}\right)\right]=-\frac{1}{4 \sigma^{2}}\left(\frac{2 \mu_{1}-\bar{X}_{1}-\bar{X}_{2}}{2 \sigma}\right) \phi\left(\frac{2 \mu_{1}-\bar{X}_{1}-\bar{X}_{2}}{2 \sigma}\right) \\
+\frac{\lambda_{3}}{24 \sigma^{4}} D^{4} \phi\left(\frac{\bar{X}_{1}+\bar{X}_{2}-2 \mu_{1}}{2 \sigma}\right) . \\
\frac{\partial^{2}}{\partial \bar{X}_{1} \partial \bar{X}_{2}}\left[e_{12}\left(\bar{X}_{1}, \bar{X}_{2}\right)\right]=-\frac{1}{4 \sigma^{2}}\left(\frac{2 \mu_{1}-\bar{X}_{1}-\bar{X}_{2}}{2 \sigma}\right) \phi\left(\frac{2 \mu_{1}-\bar{X}_{1}-\bar{X}_{2}}{2 \sigma}\right) \\
+\frac{\lambda_{3}}{24 \sigma^{4}} D^{4} \phi\left(\frac{\bar{X}_{1}+\bar{X}_{2}-2 \mu_{1}}{2 \sigma}\right) .
\end{gathered}
$$

It should be noted that at point $\left(\mu_{1}, \mu_{2}\right)$, certain equalities hold [i.e. Eq. (11) equals to Eq. (12)]. See Eq. (20) and [9]. From Eqs. (9) - (11), we expand the terms involving $D^{2}, D^{3}$ and $D^{4}$ in Chebyshev's-Hermite polynomials to get 


$$
\begin{gathered}
D^{2} \phi\left[\frac{\bar{X}_{1}+\bar{X}_{2}-2 \mu_{1}}{2 \sigma}\right]=\left\{\left[\left(\frac{\bar{X}_{1}+\bar{X}_{2}-2 \mu_{1}}{2 \sigma}\right)\right]-1\right\} \cdot\left[\frac{\bar{X}_{1}+\bar{X}_{2}-2 \mu_{1}}{2 \sigma}\right] \\
D^{3} \phi\left[\frac{\bar{X}_{1}+\bar{X}_{2}-2 \mu_{1}}{2 \sigma}\right]=\left\{\left[-\left(\frac{\bar{X}_{1}+\bar{X}_{2}-2 \mu_{1}}{2 \sigma}\right)\right]^{3}+3\left[\frac{\bar{X}_{1}+\bar{X}_{2}-2 \mu_{1}}{2 \sigma}\right]\right\} \phi\left[\frac{\bar{X}_{1}+\bar{X}_{2}-2 \mu_{1}}{2 \sigma}\right] \\
D^{4} \phi\left[\frac{\bar{X}_{1}+\bar{X}_{2}-2 \mu_{1}}{2 \sigma}\right]=\left\{\left[\frac{\bar{X}_{1}+\bar{X}_{2}-2 \mu_{1}}{2 \sigma}\right]^{4}-6\left[\frac{\bar{X}_{1}+\bar{X}_{2}-2 \mu_{1}}{2 \sigma}\right]^{2}+3\right\} \phi\left[\frac{\bar{X}_{1}+\bar{X}_{2}-2 \mu_{1}}{2 \sigma}\right]
\end{gathered}
$$

Setting $\alpha=\frac{\left(\mu_{2}-\mu_{1}\right)}{2 \sigma}$ and evaluating Eqs. (9), (10) and (11), we have

$$
\begin{gathered}
\left.e_{12}\left(\bar{X}_{1}, \bar{X}_{2}\right)\right|_{\mu_{1}, \mu_{2}}=\phi(-\alpha)+\frac{\lambda_{3}}{6 \sigma^{2}}\left[\alpha^{2}-1\right] \phi(\alpha)=m_{1} \\
\left.\frac{\partial}{\partial \bar{X}_{1}}\left[e_{12}\left(\bar{X}_{1}, \bar{X}_{2}\right)\right]\right|_{\mu_{1}, \mu_{2}}=-\frac{1}{2 \sigma} \phi(-\alpha)+\frac{\lambda_{3}}{12 \sigma^{3}}\left[3 \alpha-\alpha^{3}\right] \phi(\alpha)=m_{2} \\
\left.\frac{\partial^{2}}{\partial \bar{X}_{1}}\left[e_{12}\left(\bar{X}_{1}, \bar{X}_{2}\right)\right]\right|_{\mu_{1}, \mu_{2}}=\frac{1}{4 \sigma} \alpha \phi(-\alpha)+\frac{\lambda_{3}}{24 \sigma^{4}}\left[\alpha^{4}-6 \alpha^{2}+3\right] \phi(\alpha)=m_{3} \\
\frac{\partial^{2}}{\partial \bar{X}_{1} \bar{X}_{2}}\left[e_{12}\left(\bar{X}_{1}, \bar{X}_{2}\right)\right]=\frac{1}{4 \sigma} \alpha \Phi(-\alpha)+\frac{\lambda_{3}}{24 \sigma^{4}}\left[\alpha^{4}-6 \alpha^{2}+3\right] \phi(\alpha)=m_{3}
\end{gathered}
$$

The following equalities hold at point $\left(\mu_{1}, \mu_{2}\right)$ :

$$
\begin{aligned}
\frac{\partial}{\partial \bar{X}_{1}}\left[e_{12}\left(\bar{X}_{1}, \bar{X}_{2}\right)\right] & =\frac{\partial}{\partial X_{2}}\left[e_{12}\left(\bar{X}_{1}, \bar{X}_{2}\right)\right], \\
\frac{\partial^{2}}{\partial \bar{X}_{1}}\left[e_{12}\left(\bar{X}_{1}, \bar{X}_{2}\right)\right]=\frac{\partial^{2}}{\partial \bar{X}_{2}}\left[e_{12}\left(\bar{X}_{1}, \bar{X}_{2}\right)\right] & =\frac{\partial^{2}}{\partial \bar{X}_{1} \partial \bar{X}_{2}}\left[e_{12}\left(\bar{X}_{1}, \bar{X}_{2}\right)\right] \\
= & \frac{\partial^{2}}{\partial \bar{X}_{2} \partial \bar{X}_{1}}\left[e_{12}\left(\bar{X}_{1}, \bar{X}_{2}\right)\right]
\end{aligned}
$$

If we neglect the terms made up of partial derivatives of order higher than the second, we have,

$$
\begin{aligned}
e_{12}\left(\bar{X}_{1}, \bar{X}_{2}\right) & =m_{1}+\left[\left(\bar{X}_{1}-\mu_{1}\right)+\left(\bar{X}_{2}-\mu_{2}\right)\right] m_{2} \\
& +\frac{1}{2 !}\left[\left(\bar{X}_{1}-\mu_{1}\right)^{2}+2\left(\bar{X}_{1}-\mu_{1}\right)\left(\bar{X}_{2}-\mu_{2}\right)+\left(\bar{X}_{2}-\mu_{2}\right)^{2}\right] m_{3}+G_{2}
\end{aligned}
$$

The expansion of $e_{12}\left(\bar{X}_{1}, \bar{X}_{2}\right)$ is obtained in a similar form when $\bar{X}_{1} \geq \bar{X}_{2}$ as

$$
\begin{aligned}
e_{12}\left(\bar{X}_{1}, \bar{X}_{2}\right) & =m_{1}^{\prime}+\left[\left(\bar{X}_{1}-\mu_{1}\right)+\left(\bar{X}_{2}-\mu_{2}\right)\right] m_{2}^{\prime} \\
& +\frac{1}{2 !}\left[\left(\bar{X}_{1}-\mu_{1}\right)^{2}+2\left(\bar{X}_{1}-\mu_{1}\right)\left(\bar{X}_{2}-\mu_{2}\right)+\left(\bar{X}_{2}-\mu_{2}\right)^{2}\right] m_{3}^{\prime}+G_{2}^{\prime}
\end{aligned}
$$

where 


$$
\begin{gathered}
m_{1}^{\prime}=\phi(\alpha)-\frac{\lambda_{3}}{6 \sigma^{2}}\left[\alpha^{2}-1\right] \phi(\alpha) \\
m_{2}^{\prime}=\frac{1}{2 \sigma} \phi(\alpha)-\frac{\lambda_{3}}{12 \sigma^{2}}\left[3 \alpha-\alpha^{3}\right] \phi(\alpha) \\
m_{3}^{\prime}=\frac{1}{4 \sigma^{2}} \alpha \phi(\alpha)-\frac{\lambda_{3}}{24 \sigma^{2}}\left[\alpha^{4}-6 \alpha^{2}+3\right] \phi(\alpha) .
\end{gathered}
$$

\subsection{Approximate Mean and Variance of Conditional Probability of Misclassification}

We obtain the approximate mean and variance of the conditional probability of misclassification from Eqs. (21) and (22). Let $(X, Y)$ be a two-dimensional random variable.

Suppose $E(X)=\mu_{x}, E(Y)=\mu_{y}$ and $\mathrm{V}(X)=\sigma_{x}^{2}, V(Y)=\sigma_{y}^{2}$.

Set $Z=B(X, Y)$ and assume that at point $\left(\mu_{x}, \mu_{y}\right)$, various derivatives of $B$ exist so that

$$
\begin{gathered}
E(Z)=B\left(\mu_{x}, \mu_{y}\right)+\frac{\partial^{2} B}{\partial X^{2}}+\frac{\partial^{2} B}{\partial Y^{2}} \partial_{y}^{2} \\
V(Z)=\left[\frac{\partial B}{\partial X}\right]^{2} \sigma_{x}^{2}+\left[\frac{\partial H}{\partial Y}\right]^{2} \sigma_{y}^{2}
\end{gathered}
$$

where all partial derivatives are evaluated at the point $\left(\mu_{x}, \mu_{y}\right)$. See [2]

$$
\text { Let } \begin{aligned}
F & =\left(\bar{X}_{1}-\mu_{1}\right)+\left(\bar{X}_{2}-\mu_{2}\right) \\
& =\left(\bar{X}_{1}+\bar{X}_{2}\right)-\left(\mu_{1}+\mu_{2}\right) .
\end{aligned}
$$

Also, let $\bar{X}_{1}<\bar{X}_{2}$, so that from Eq. (22),

$$
e_{12}\left(\overline{X_{1}}, \overline{X_{2}}\right)=m_{1}+m_{2} F+m_{3} F^{2}+G_{2}
$$

where

$$
\left.\begin{array}{c}
m_{3}^{\prime}=\frac{m_{2}}{2} \\
E(F)=0, V(F)=\sigma^{2}\left(\frac{1}{n_{1}}+\frac{1}{n_{2}}\right) \\
V\left(F^{2}\right)=E\left(F^{4}\right)-E^{2}\left(F^{2}\right) \\
=3 \sigma^{4}\left[\frac{\sigma^{2}}{n_{1}}+\frac{\sigma^{2}}{n_{2}}\right]^{2}-\left[\frac{\sigma^{2}}{n_{1}}+\frac{\sigma^{2}}{n_{2}}\right]^{2}
\end{array}\right\}
$$

See [10].

The approximate mean of $e_{12}\left(\bar{X}_{1}, \bar{X}_{2}\right)$ is given by

$$
\begin{aligned}
E\left[e_{12}\left(\bar{X}_{1}, \bar{X}_{2}\right)\right] & \approx m_{1}+m_{2} E(F)+m_{3}^{\prime} E\left(F^{2}\right) \\
& =m_{1}+m_{3}^{\prime}\left[\frac{\sigma^{2}}{n_{1}}+\frac{\sigma^{2}}{n_{2}}\right]
\end{aligned}
$$


and the approximate variance of $e_{12}\left(\bar{X}_{1}, \bar{X}_{2}\right)$ is given by

$$
\begin{aligned}
V\left[e_{12}\left(\bar{X}_{1}, \bar{X}_{2}\right)\right] \approx & m_{2}^{2} E\left(F^{2}\right)+\left(m_{3}^{\prime}\right)^{2} E\left[F^{2}-\sigma^{2}\left(\frac{1}{n_{1}}+\frac{1}{n_{2}}\right)\right]^{2} \\
& +2 m_{2} m_{3}^{\prime} E\left[F\left(F^{2}-\sigma^{2}\left[\frac{1}{n_{1}}+\frac{1}{n_{2}}\right]\right)\right] \\
= & m_{2}^{2} E\left(F^{2}\right)+\left(m_{3}^{\prime}\right)^{2} V\left(F^{2}\right)+2 m_{2} m_{3}^{\prime} E\left(F^{3}\right) \\
& -2 m_{2} m_{3}^{\prime}\left[\frac{\sigma^{2}}{n_{1}}+\frac{\sigma^{2}}{n_{2}}\right] E(F) .
\end{aligned}
$$

Using the results in Eq. (30), we have

$$
\begin{aligned}
V\left[e_{12}\left(\bar{X}_{1}, \bar{X}_{2}\right)\right] \approx & m_{2}^{2}\left[\frac{\sigma^{2}}{n_{1}}+\frac{\sigma^{2}}{n_{2}}\right]+\left(m_{3}^{\prime}\right)^{2}\left[3 \sigma^{4}\left(\frac{1}{n_{1}}+\frac{1}{n_{2}}\right)^{2}\right] \\
& -\sigma^{4}\left(\frac{1}{n_{1}}+\frac{1}{n_{2}}\right)^{2}+2 m_{1} m_{3}^{\prime} \lambda_{3}\left(\frac{1}{n_{1}^{2}}+\frac{1}{n_{2}^{2}}\right) \\
\approx & m_{2}^{2}\left[\frac{\sigma^{2}}{n_{1}}+\frac{\sigma^{2}}{n_{2}}\right]+\left(m_{3}^{\prime}\right)^{2} 2 \sigma^{4}\left[\frac{1}{n_{1}}+\frac{1}{n_{2}}\right]^{2} \\
& +2 m_{2} m_{3}^{\prime} \lambda_{3}\left[\frac{1}{n_{1}^{2}}+\frac{1}{n_{2}^{2}}\right], \bar{X}_{1}<\bar{X}_{2}
\end{aligned}
$$

Hence,

$$
\begin{aligned}
E\left[e_{12}\left(\bar{X}_{1}, \bar{X}_{2}\right)\right] & \approx m_{1}+m_{3}^{\prime} \sigma^{2}\left[\frac{1}{n_{1}}+\frac{1}{n_{2}}\right], \bar{X}_{1}<\bar{X}_{2} \\
& \approx m_{1}^{\prime \prime}+m_{3}^{\prime \prime} \sigma^{2}\left[\frac{1}{n_{1}}+\frac{1}{n_{2}}\right], \bar{X}_{1} \geq \bar{X}_{2}
\end{aligned}
$$

where $m_{3}{ }^{\prime \prime}=\frac{m_{3}^{\prime}}{2}$, and

$$
\begin{aligned}
& V\left[e_{12}\left(\bar{X}_{1}, \bar{X}_{2}\right)\right] \approx m_{2}^{2}\left(\frac{\sigma^{2}}{n_{1}}+\frac{\sigma^{2}}{n_{2}}\right)+\left(m_{3}^{\prime}\right)^{2} \sigma^{4}\left[\frac{1}{n_{1}}+\frac{1}{n_{2}}\right]^{2} \\
&+2 m_{2} m_{3}^{\prime} \lambda_{3}\left[\frac{1}{n_{1}^{2}}+\frac{1}{n_{2}^{2}}\right], \quad \bar{X}_{1}<\bar{X}_{2} \\
& \approx\left(m_{2}^{\prime}\right)^{2}\left(\frac{\sigma^{2}}{n_{1}}+\frac{\sigma^{2}}{n_{2}}\right)+\left(m_{3}^{\prime \prime}\right)^{2} 2 \sigma^{4}\left[\frac{1}{n_{1}}+\frac{1}{n_{2}}\right]^{2} \\
&+2 m_{2}^{\prime} m_{3}^{\prime \prime} \lambda_{3}\left[\frac{1}{n_{1}^{2}}+\frac{1}{n_{2}^{2}}\right], \quad \bar{X}_{1} \geq \bar{X}_{2} .
\end{aligned}
$$

The asymptotic distribution of $e_{12}\left(\bar{X}_{1}, \bar{X}_{2}\right)$ is considered since the problem is symmetric with respect to the error. As $n_{1}$ and $n_{2}$ become large, the distribution of 


$$
y=\frac{e_{12}\left(\bar{X}_{1}, \bar{X}_{2}\right)-E\left(e_{12}\left\{\bar{X}_{1}, \bar{X}_{2}\right\}\right)}{\left[V\left\{e_{12}\left(\bar{X}_{1}, \bar{X}_{2}\right)\right\}\right]^{1 / 2}}
$$

is approximately normal with mean 0 and variance 1 .

Ignoring terms of order $\frac{1}{n_{1}^{2}}, \frac{1}{n_{2}^{2}}$ and $\frac{1}{n_{1} n_{2}}$ in the mean- variance approximation [see Eqs. (32) and (33)], we have

$$
\begin{aligned}
\operatorname{Pr}\left\{e_{12}\left(\bar{X}_{1}, \bar{X}_{2}\right)<u\right\} & =\operatorname{Pr}\left\{\frac{e_{12}\left(\bar{X}_{1}, \bar{X}_{2}\right)-\left[k_{1}+\left(k_{3}^{\prime}\right)^{2}\left(\frac{\sigma^{2}}{n_{1}}+\frac{\sigma^{2}}{n_{2}}\right)\right]}{\left[k_{2}^{2}\left(\frac{\sigma^{2}}{n_{1}}+\frac{\sigma^{2}}{n_{2}}\right)\right]^{1 / 2}}<\frac{u-\left[k_{1}+\left(k_{3}^{\prime}\right)^{2}\left(\frac{\sigma^{2}}{n_{1}}+\frac{\sigma^{2}}{n_{2}}\right)\right]}{\left[k_{2}^{2}\left(\frac{\sigma^{2}}{n_{1}}+\frac{\sigma^{2}}{n_{2}}\right)\right]^{1 / 2}}\right\} \\
& =\Phi\left[\frac{u-\left[k_{1}+\left(k_{3}^{\prime}\right)^{2}\left(\frac{\sigma^{2}}{n_{1}}+\frac{\sigma^{2}}{n_{2}}\right)\right]}{\left[k_{2}^{2}\left(\frac{\sigma^{2}}{n_{1}}+\frac{\sigma^{2}}{n_{2}}\right)\right]^{1 / 2}}\right] \text { when } \bar{X}_{1}<\bar{X}_{2}
\end{aligned}
$$

and

$$
\operatorname{Pr}\left\{e_{12}\left(\bar{X}_{1}, \bar{X}_{2}\right)<u\right\}=\Phi\left[\frac{u-\left[k_{1}^{\prime}+\left(k_{3}^{\prime \prime}\right)^{2}\left(\frac{\sigma^{2}}{n_{1}}+\frac{\sigma^{2}}{n_{2}}\right)\right]}{\left(k_{3}^{\prime}\right)^{2}\left[\left(\frac{\sigma^{2}}{n_{1}}+\frac{\sigma^{2}}{n_{2}}\right)\right]^{1 / 2}}\right] \text { when } \bar{X}_{1} \geq \bar{X}_{2}
$$

\section{Conclusion}

The asymptotic distribution of probabilities of misclassification for Edgeworth Series Distribution using Taylor's series expansion has been derived in this study. The conditional probability of misclassification as a function of $\bar{X}_{1}$ and $\overline{X_{2}}$ has been expanded in a Taylor series in two random variables in the region of $E\left(\bar{X}_{1}\right)=\mu_{1}, E\left(\overline{X_{2}}\right)=\mu_{2}$. The approximate mean and variance of the conditional probabilities of misclassification were subsequently evaluated. It was established that as $n_{1}$ and $n_{2}$ become large, the distribution of $\frac{e_{12}-E\left(e_{12}\right)}{\left[V\left\{e_{12}\right\}\right]^{1 / 2}}$ is approximately normal with mean 0 and variance unity.

\section{References}

[1] Babu, G. J. and Singh, K. (1985). Edgeworth Expansions for Sampling without Replacement from Finite Populations. Journal of Multivariate Analysis, 17: 261-278.

[2] Bhuyan, C. K. (2010), Probability Theory and Statistical Inference, $1^{\text {st }}$ ed. New Central Book Agency (Ltd), London.
[3] Does, R. J. M. M., Helmers, R. and Klaassen, C. A. J. (1987). Approximating the Distribution of Greenwood's Statistics. Statistica Neerlandica, 42: 153-162.

[4] Ekezie, D. D. and Onyeagu, S. I. (2013). Comparison of Seven Asymptotic Expansion for the Sample Linear Discriminant. Function, Canadian Journal of Computations in Mathematics, Natural Sciences, Engineering and Medicine, 4 (1): 93-115.

[5] Ghosh, K. and Jammalamadaka, S. R. (1998). Small Sample Approximations for Spacing Statistics. Journal of Statistical Planning and Inference, 69: 245-261.

[6] Goncalves, S. and Meddahi, N. (2009), Bootstrap Realized Volatility. Econometrica, 77 (1): 283-306.

[7] Gudhinder, K. and Paul, R. (2012). Edgeworth Expansions for GEL Estimator. Journal of Multivariate Analysis, 106: 118146.

[8] Kendall, M. G. and Stuart, A. (1958). The Advanced Theory of Statistics, Hafner: New York.

[9] Kocherlakota, S. and Chinganda, E. F. (1978). Robustness of the Linear Discriminant Function to Non-normality: Edgeworth Series Distribution. Journal of Statistical Planning and Inference, 2: 79-91.

[10] Meyer, P. L. (1966). Introductory Probability and Statistical Applications. Addison-Wesley: Reading, Mass. 
[11] Muhammad, N. (2017). On the Edgeworth Type Expansions for the Distribution of Extreme Spacing Statistics. Pakistan Journal of Statistics, 33 (2): 117-128.

[12] Peter, H. (1997). The Bootstrap and Edgeworth Expansions. Springer Series in Statistics, 145.

[13] Rani, B. (2010). Edgeworth Expansion and Saddle Point Approximation for Discrete Data with Application to Chance Game. M. Sc. Thesis, University of Linnaeus, School of Computer, Physics and Mathematics, 1-39.

[14] Ruby, C. W. (2010). A Bayesian Edgeworth Expansion by Stein's Identity. Bayesian Analysis, 5 (4): 741-764.

[15] Ulrich, H. and Bezirgen, V. (2015). Validity of Edgeworth Expansions for Realized Volatility Estimators. CREATES
Research Papers 2015-21, Centre for Research in Econometrics Analysis of Time Series, Department of Economics and Business Aarhus University, Denmark, 1-31.

[16] Wu, X. and Wang, S. (2011). Maximum Entropy Approximations for Asymptotic Distributions of Smooth Functions of Sample. Scandinavian Journal of Statistics, 38: $130-146$.

[17] Yonatan, R. and Monika, P. (2014). Edgeworth Expansion Based Model for the Convolutional Noise pdf. Mathematical Problems in Engineering, 20 (14): 1-19.

[18] Zarkar, K., Alenxader, M. \& Henning, S. (2016). Mode and Edgeworth Expansion for the Ewens Distribution and the Stirling Number., Journal of Integer Sequences, 19: 1-7. 